\title{
An in vitro - agent based modelling approach to optimisation of culture medium for generating muscle cells
}

\author{
David Hardman ${ }^{1}$, Katharina Hennig ${ }^{2}$, Edgar Gomes $^{2}$, William Roman ${ }^{2}$, Miguel O. Bernabeu ${ }^{1,3}$ \\ 1 Centre for Medical Informatics, Usher Institute, The University of Edinburgh, \\ Edinburgh EH16 4UX, United Kingdom \\ 2 Instituto de Medicina Molecular, Faculdade de Medicina, Universidade de Lisboa, \\ Avenida Professor Egas Moniz, 1649-028, Lisboa, Portugal \\ 3 The Bayes Centre, The University of Edinburgh, Edinburgh EH8 9BT, United \\ Kingdom
}

\begin{abstract}
Many of the protocols in contemporary tissue engineering remain insufficiently optimised. Methodologies for culturing the complex structures of muscle tissue are particularly lacking, both in terms of quality and quantity of mature cells. Here, we analyse images from in vitro experimentation to quantify the effects of the composition of culture media on mouse-derived myoblast behaviour and myotube cell quality. Computational modelling was then used to predict the optimum range of media compositions for culturing. We define a metric of uniformity of myonuclei distribution as an early indicator of cell quality and difference in myonuclei density over time as an indicator of cell quantity. Analysis of live and static images of muscle cell differentiation revealed that changes in culture media result in significant changes in indicators of cell quantity and quality as well as changes in myoblast migratory behaviour. By describing cell behaviours as a set of functions of media composition we designed a model for predicting cell quality. Cell behaviours were taken directly from experimental images or inferred using Approximate Bayesian Computation and applied as inputs to an agent-based model of cell differentiation which predicted cell quality indicators. Our results suggest that culturing muscle cells in a neural cell differentiation medium reduces the quantity of cell fusion, but does not diminish cell quality. We also show that, while high concentrations of serum are detrimental to cell development, increasing serum concentration raises the total amount of myoblast fusion. This implies a trade-off between the quantity and quality of cells produced when choosing a culture medium. Our model provided a good prediction of experimental results for media with $5 \%$ serum provided the background cell proliferation rate was known.
\end{abstract}

\section{Introduction}

Organ-on-a-chip technologies aim to mimic human tissue structure in vitro in order to recapitulate organ function. To imitate physiological, mechanical, and biochemical properties of the native tissue environment, the system relies on a combination of microfluidics, biomaterials and cell cultures 1 . Various organ-on-chip platforms successfully mimic organ systems such as lung 2, heart 3], and liver 4] while other biomimetic in vitro systems on a chip remain challenging.

One major limitation in muscle-on-a-chip technology lies in reproducing the complex hierarchical structure of muscle tissue in vitro [5. To form contractile myotubes, distinct cell sources are required. Currently, the most relevant in vitro myogenesis model employs primary myoblasts [6, which differentiate into multinucleated myotubes.

Successful motor neuron innervation may affect muscle differentiation and contractility strength beyond synapse formation[7]. Neuronal derived factors, such as agrin and neuregulin- 1 , have been shown to increase muscle maturation, while at the same time, promoting neuromuscular junction (NMJ) formation.

Therefore, successfully mimicking physiological processes in vitro depends upon the harmonious co-culturing of multiple cell types. Meeting the combined requirements for co-culturing increases 
experimental complexity, requiring new strategies in process optimisation 8. A robust quantification and optimisation of experimental parameters in cell culturing processes can maximise the quality and yield of the final product while reducing costs and experimental time and enhancing experimental reproducibility.

Successful in vitro myogenesis is determined by the quality and quantity of the mature cells. Healthy muscle cells are long and multinucleated with cell nuclei evenly distributed throughout the periphery of the fibre. Uneven or centralised distribution of nuclei is often linked to a disease state or improper maturation $[9],[10$.

To reflect the functional and structural characteristics of native skeletal muscle in vivo, the properties of the artificial microenvironment need to be fine-tuned. A crucial initial step is optimisation of cell culture medium to ensure cell survival, growth and maturation. An appropriate growth medium is dependent upon the base medium, serum percentage and source, and the addition of soluble factors. For instance, the fusion of primary myoblasts into multinucleated myotubes is initiated upon serum starvation and promoted by the addition of neuronal-derived factors such as agrin 11. On the other hand, to ensure proper motor neuron differentiation, neuronal media (such as N2B27) are supplemented with factors (e.g. BDNF, GDNF), which steer cell linage, and are usually serum-free to limit batch-to-batch as well as source-to-source variations in hormone, growth factor, and antibody content 12 .

Screening the media conditions that facilitate optimal muscle cell formation is necessary but complex. To do so, in vitro experiments have been an invaluable tool for exploring cell culture environments 13 but are expensive, time consuming and generally provide sparse data points for analysis. Use of high throughput systems can reduce the amount and cost of trials required but are hampered when critical parameters are poorly understood. Numerical models of cell and tissue behaviours [14] present fast, lowcost methods for simulating in vitro experiments but require thorough calibration against experimental results to establish confidence in predictions. Here, we apply a combined in vitro-in silico approach in which experimental data is used to calibrate and refine a numerical model which can then be used to inform future in vitro experiments such as myogenesis, NMJ formation or disease modelling in an iterative fashion. This allows us to efficiently predict cell and tissue behaviours over all relevant parameter values for a given experiment.

Agent based models (ABM) have gained increasing popularity among numerical models used to compliment cell and tissue engineering, thanks to their versatility and ability to recapitulate higher level emerging behaviours from sets of simple rules based upon known constraints 15]. Systematic refinement of the rules governing cell behaviours in ABM allows us to gain insight into the underlying mechanisms which may cause any deviations.

In this study, we focus on optimising the production of skeletal muscle by designing an experimental protocol that maximises muscle cell quality. The goal was to define an optimal range of media conditions, namely base medium and serum content, in preparation for co-culturing myotubes with neurons to create innervated muscle tissue.

We conducted two sets of in vitro experiments, firstly observing myotube differentiation and maturation under a range of serum concentrations $(0,2,5$ and $10 \%)$ and, secondly, distinct ratios of $2 \%$ serum muscle differentiation medium to neuronal medium $(1: 0 ; 1: 1,0: 1)$. We then determined the effects of changing media conditions on muscle cell yield, quality and experimental efficiency. In this study, we have identified the uniformity of spatial distribution of nuclei and the number of myoblast fusion events as novel quality indicators with which to assess experimental success.

Our initial experiments show that changes in both serum concentration and proportion of neuronal medium result in significant changes in cell behaviours during the early stages of cell differentiation, which ultimately affect muscle maturation. Strikingly, metrics of early myoblast behaviour obtained from live images show that changes in media composition significantly alter the speed and angular motion of myoblast cells as well as total incidences of fusion events, resulting in altered spatial distribution of myonuclei in matured myotubes. From these findings we hypothesise that changes in myoblast and myotube behaviour in the early stages of cell differentiation, due to the composition of cell differentiation media, relate to the quality and quantity of cells produced in vitro.

Motivated by these findings, we present a workflow for predicting final muscle cell quality by applying metrics of cell behaviours to an ABM of cell fusion and nuclei force balance with metrics of cell quality indicators as an output. Key metrics of cell behaviours were observed from in vitro imaging and 
inferred using Approximate Bayesian Computation (ABC). By extrapolating these metrics from discrete experiments, we describe cell behaviours as a function of media composition which, when used as an input for a calibrated ABM, can produce in silico experiments which predict the range of media conditions in which muscle cells can thrive and highlight the optimum conditions for culturing muscle cells.

\section{Methods}

\section{In vitro primary myotube culture}

In vitro myotubes were cultured from primary mice myoblasts as described previously 11. Shortly, hind limb muscles were isolated from 5-7 day old mice pubs, minced and digested for 1.5 hours at $37^{\circ} \mathrm{C}$ using $0.5 \mathrm{mg} / \mathrm{ml}$ collagenase (Sigma) and $3.5 \mathrm{mg} / \mathrm{ml}$ dispase (Roche) in PBS. Subsequently, filtered cell suspension was plated in IMDM (Invitrogen) for $4 \mathrm{~h}$ in the incubator $\left(37^{\circ} \mathrm{C}, 5 \% \mathrm{CO} 2\right)$. To purify cells from fibroblasts and other contaminating cell types, only non-adherent myoblasts were collected and centrifuged. Cells were resuspended in growth medium (IMDM $+20 \% \mathrm{FBS}+1 \%$ Chick Embryo Extract $+1 \%$ Penicillin/streptomycin) and plated onto 1:100 matrigel (RD) coated ibidi dishes. After 4 days, medium was changed to trigger differentiation. Depending on the experimental condition, standard muscle differentiation medium (IMDM + 1\% Penicillin-Streptomycin) and neuronal medium (N2B27 medium: 50\% DMEM-F12, 50\% Neurobasal +1X N2 + 1X B27 + 50 $\mu \mathrm{M} \beta$-Mercaptoethanol $+1 \%$ Penicillin-Streptomycin) were mixed 1:0, 1:1 or 0:1, containing 0,2, 5 or $10 \%$ horse serum. After 1 day of differentiation, a thick layer of 1:1 matrigel was added on top of forming myotubes and 100ng/ml agrin was added to the culture medium. Cells were cultured up to 7 days at $37^{\circ} \mathrm{C} / 5 \% \mathrm{CO} 2$.

\section{Live imaging}

Live imaging was performed using an inverted microscope (Zeiss Cell Observer SD or Zeiss Cell observer) in widefield mode, using a 20x phase air objective (Plan-Apochromat Ph2 NA 0.80 or EC Plan-Neofluar Ph 2 NA 0.50, respectively). Cells were imaged 2 hours after adding the respective differentiation medium. $3 \times 3$ tiles with $10 \%$ overlap with $2 \times 2$ binning were taken every 5 minutes for a minimum of 12 hours. Images were stitched using Zen Blue software and stacked into 30 minutes videos for further quantitative analysis.

\section{Immunostaining and static imaging}

Cells were washed once with PBS and fixed in 4\% PFA for 10 minutes at RT. Cells were permeabilized $(\mathrm{PBS}+0.5 \%$ Triton) for 5 minutes and blocked in blocking buffer $(10 \%$ in Goat serum in PBS $+5 \%$ BSA) for 1 hour at room temperature. Primary antibodies (1:200; Anti- $\alpha$-Actinin (Sarcomeric) mouse monoclonal antibody, Sigma Aldrich and A7732) were diluted in blocking buffer containing $0.1 \%$ saponine and cells were incubated at $4^{\circ} \mathrm{C}$ over night. Dishes were washed $2 \mathrm{x}$ for 5 minutes in PBS under agitation. Secondary antibodies (1:400; Goat anti-Mouse IgG Alexa Fluor 555, Thermo Fisher Scientific, A21424; Goat anti-Rabbit IgG Alexa Fluor 647, Thermo Fisher Scientific, A21245) in blocking buffer were incubated in presence of DAPI $(100 \mu \mathrm{g} / \mathrm{ml})$ for 1 hour. Dishes were washed twice, 200ul Fluoromount-G (Invitrogen) was added on top of cells. Samples were stored at $4^{\circ} \mathrm{C}$. Dishes were imaged with an inverted fluorescent microscope (Zeiss Cell observer) using a 40x phase air objective (EC Plan-NeoFluar NA 0.75). z-stacks of $1 \mu \mathrm{m}$ were taken as $3 \times 3$ tiles with $10 \%$ overlap and $1 \times 1$ binning. Subsequently, imaged were stitched in Zen Blue.

\section{Image processing and quantification}

Mononucleated myoblast cells were identified by cell area and segmented from live brightfield images (Figure 1 a) through a semi-automated process using an in-house MATLAB code. Cell centroids were tracked over two-hour periods providing data for calculating metrics of cell motion. Absolute values of the shortest distance between cells in consecutive frames were used to calculate myoblast speed. Angular 
(a)

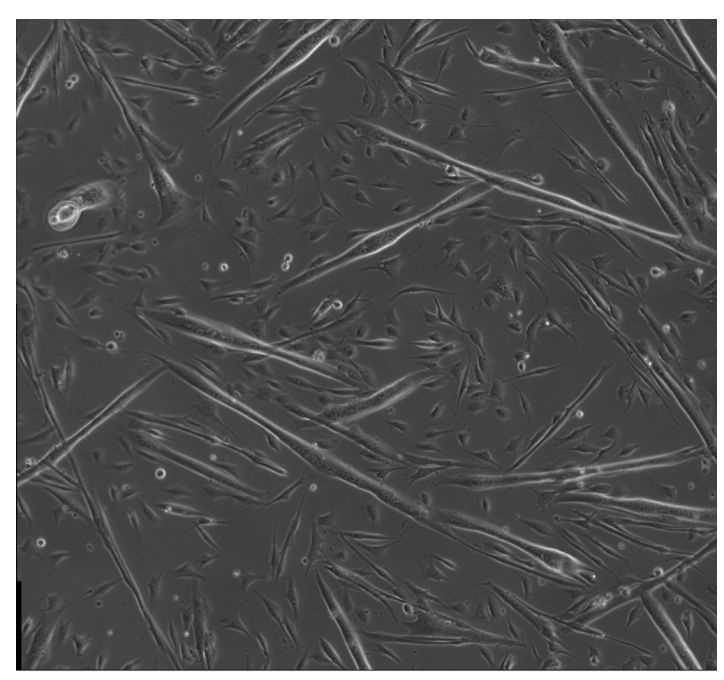

(b)

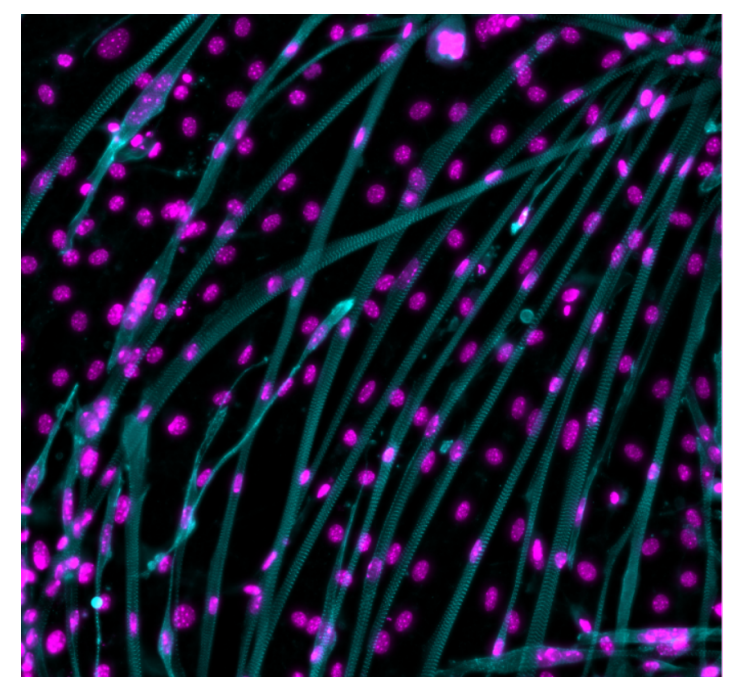

Figure 1. Examples of (a) a live, brightfield, image of myoblasts and myotubes on day 1 of differentiation (b) a static image of myotubes with actin cytoskeleton stained with (cyan)a-actinin and nuclei stained with (magenta) SiR-DNA

velocity was calculated from clockwise or anti-clockwise change in angle of cell motion between frames. Applying Rayleigh-Moore tests to mean angular velocities showed that global cell motion showed no directional preference for all trials. The standard deviation of angular velocity, doubled to account for directionality, was applied as a metric of angular variation over time. Cell proliferation rate was determined by manual detection of cell divisions in ImageJ over a two-hour period divided by the number of myoblast cells segmented in the first frame, giving a metric of chance of division per cell per minute. The number of myotube and myoblast nuclei per $\mathrm{mm}^{2}$ were identified using MATLAB (R2019a)(MathWorks,Natick,MA) from images with cell nuclei and actin cytoskeleton staining (Figure 1 b). Myonuclei distribution was measured in MATLAB by randomly selecting myotubes, manually labelling nuclei and calculating the distance between neighbouring nuclei. Estimated mean myotube width and percentage of myotubes with actin striations were also recorded as potential indicators of cell quality, but not significant differences were found between measured experiments (Data not shown). MATLAB code for segmentation and calculation of metrics is available on Github(https://github.com/dhardma2/MyoChip).

\section{Agent-based model}

NetLogo software (Wilensky, U. (1999). NetLogo. (http://ccl.northwestern.edu/netlogo/. Center for Connected Learning and Computer-Based Modeling, Northwestern University. Evanston, IL.) was used to create the agent-based model as described below. Code is available on Github(https: //github.com/dhardma2/MyoChip). Approximate Bayesian computation methods used to infer cell behaviours were conducted using pyABC $[16$.

\section{Statistical analysis}

The Wilcoxon signed-rank test was applied to assess mean distance between myonuclei and two-sample t-tests were applied for all other data sets below unless otherwise stated. Statistical analysis was conducted in MATLAB. 
(a)

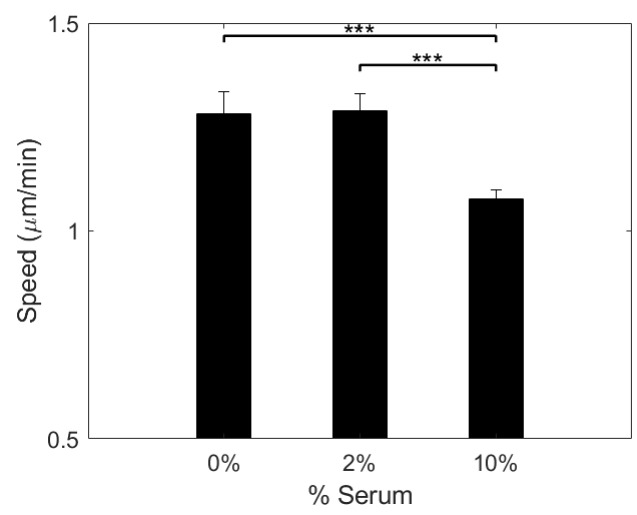

(c)

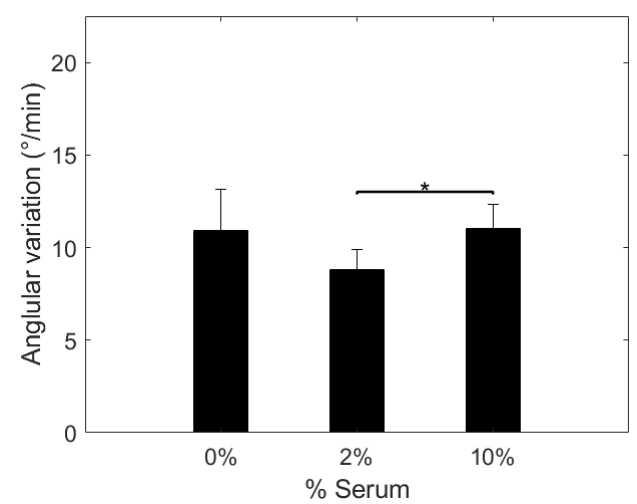

(b)

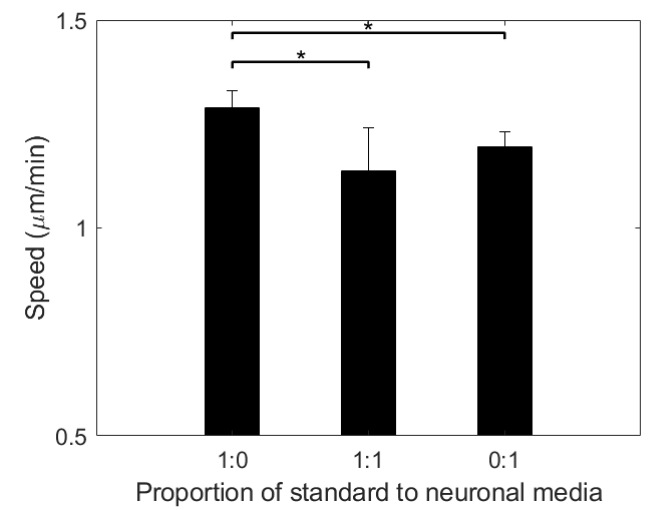

(d)

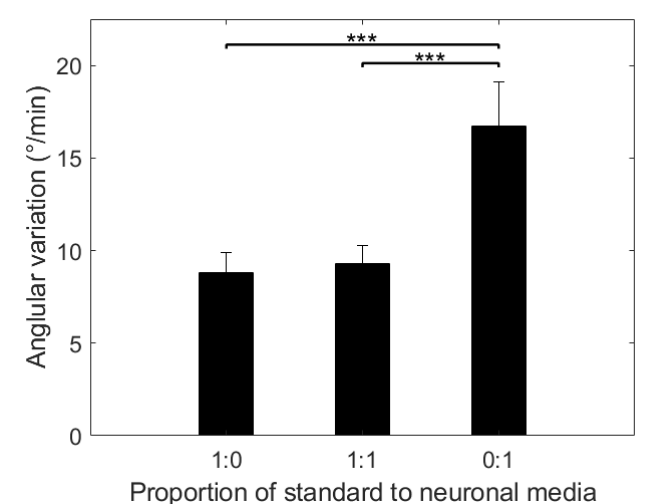

Figure 2. Metrics of myoblast behaviour from live images of cells in different media compositions. Myoblast speed and standard deviation of angular velocity in differentiation media with (a, c) varying serum concentrations and no neuronal medium and $(b, d)$ varying proportions of neuronal medium and a $2 \%$ serum concentration.

\section{Results}

Composition of differentiation media produces significant changes in locomotion behaviour of mono-nucleated myoblasts during early stage of muscle differentiation

To evaluate the effect of distinct co-culture media, we first extracted quantitative parameters that describe the migratory behaviour of mono-nucleated myoblasts within early stage (Day 0-1) of muscle differentiation. To do so, cells were subjected to either distinct serum concentrations (muscle differentiation medium supplemented with $0 \%, 2 \%$, or $10 \%$ serum) or different media mixtures (muscle differentiation medium with $2 \%$ serum mixed with neuronal medium 1:0, 1:1 or 0:0). Live images of cells during day 0-1 after the application of different media show significant and non-linear differences in metrics of myoblast migration speed and the distribution of cell angular velocities (Figure 2 (a-d)).

For serum concentrations between 0-2\%, mono-nucleated cells moved at similar speeds. Increasing serum concentration from $2 \%$ to $10 \%$ resulted in a significant reduction in cell speed (Figure 2(a)). There was also evidence of a modest dip in the deviation of angular velocity as serum concentration is increased from $0-10 \%$ (Figure 2(c)).

Concerning distinct muscle-neuron media mixtures, a decrease in cell speed was observed between cells differentiated in muscle differentiation medium and in a 1:1 mixture of muscle and neuronal differentiation

138 
medium (Figure 2(b)), however, there was no significant change in angular velocity (Figure 2(d)). Cell migration speed of myoblasts cultured in either 1:1 media mixture or 100\% neuronal medium remained similar, while the standard deviation in angular velocity increased significantly. This indicates that varying the differentiation media composition produces changes in the migratory behaviour of myoblast cells. Changes in serum percentage and the proportion of neuronal medium affect metrics of these cell behaviours in different ways.

\section{Composition of differentiation media produces changes in early-stage indica- tors of muscle cell quality}

During myogenesis, mono-nucleated myoblasts fuse to form multinucleated myotubes. To address if early myoblast migratory behaviour affects cell fusion and myotube maturation, we extracted metrics of myotube growth throughout the time-course of differentiation (day 0 - day 7 ). The number of myoblast-myotube fusion events was quantified by comparing the difference in the number of myonuclei per $\mathrm{mm}^{2}$ between days 0 and 5 of the application of distinct differentiation media. Increasing the serum concentration (Figure 3 a) from $0 \%$ to $2 \%$ led to a significant (464 more nuclei $/ \mathrm{mm}^{2}$ ) increase in the number of fusion events for cells cultured in muscle differentiation media. A further increase to $10 \%$ serum concentration resulted in a reduction in the quantity of fusion events (559 less nuclei $/ \mathrm{mm}^{2}$ ) to a level slightly lower than with no serum. This suggests an optimal serum concentration to promote muscle differentiation in vitro.

A significant drop in the number of fusion events (567 less nuclei $/ \mathrm{mm}^{2}$ ) was observed between experiments cultured in muscle differentiation media and a 1:1 mixture of muscle and neuronal media (Figure 3). Fusion events remained at a similarly low level in experiments with $100 \%$ neuronal medium, demonstrating the existence of an optimal base medium for myotube formation.

One hallmark of mature myotubes is the presence of multiple myonuclei, located at the cell periphery, uniformly distributed throughout the fibre. Uniformity of myonuclei distribution was evaluated using the coefficient of variation, calculated by dividing the standard deviation of distances between nuclei in a cell by the mean distance between nuclei in the respective cell. A lower coefficient of variation indicates greater uniformity. Applying spatial uniformity of nuclei as an indicator of quality assumes that nuclei are spread sufficiently far apart. As the coefficient of variation does not provide information on distances between nuclei, the mean distance between myonuclei was also recorded.

Increasing the concentration of serum in differentiation medium resulted in a gradual increase in the mean distance between myonuclei (Figure 3r) but, while there is some evidence of a reduction in the range of coefficient of variation, there was no significant change in uniformity (Figure 3p) of nuclei distribution.

Increasing the proportion of neuronal medium lead to an increase in mean distance between myonuclei (Figure3d). Experiments with 100\% neuronal medium and a 1:1 mixture of neuronal to muscle differentiation media exhibited a lower coefficient of variation, and therefore more uniform distribution, than muscle differentiation medium alone (Figure $3 \mathrm{f}$ ).

\section{Calibration of an in-vitro / agent-based model workflow for prediction of cell quality from early-stage cell behaviours.}

Our results (Figure 3) indicate that final quantity of myotube nuclei produced and the spatial uniformity of myonuclei within a cell, an indicator of cell quality, are dependent upon the composition of the culture media and that varying individual components of the media (in this case, percentage of horse serum and proportion of neuronal medium) affects these quality indicators in different ways. Following from this, we sought to explore the effects of adjusting concentrations both media components with the aim of predicting a range of optimal media conditions for culturing muscle cells and co-culturing muscle and neuron cells.

Having observed changes in myoblast behaviour (Figure 22) upon changing single parameters of differentiation media composition, we designed an ABM with distributions of cell behaviour metrics taken from in-vitro experimental results as inputs and cell quality indicators as an output. An overview of the

\section{0}

161

162 163 164 165 166 167 168 169 170 171 172 173 174 175 176 177 178 179 180 181 182 
(a)

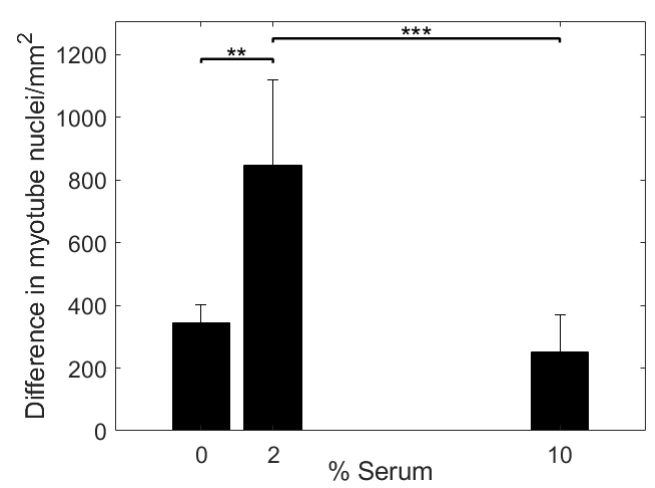

(c)

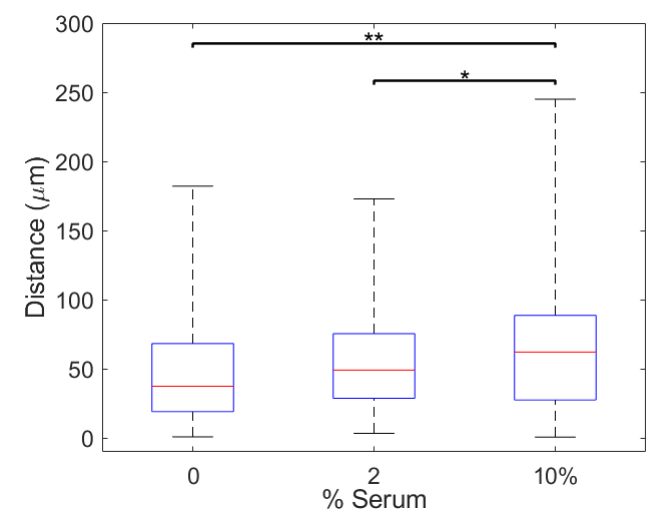

(e)

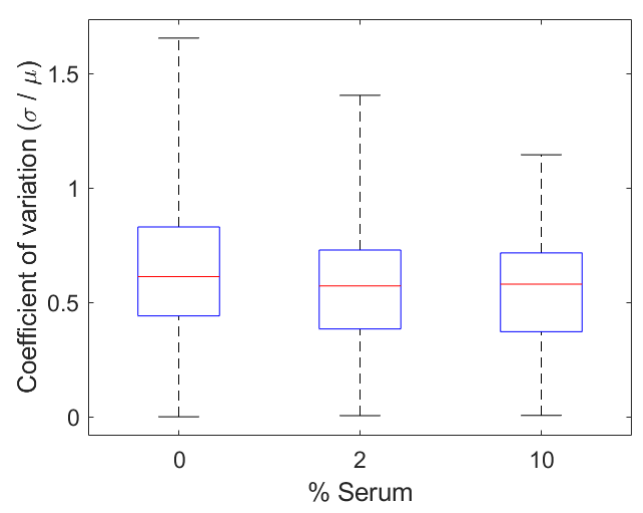

(b)

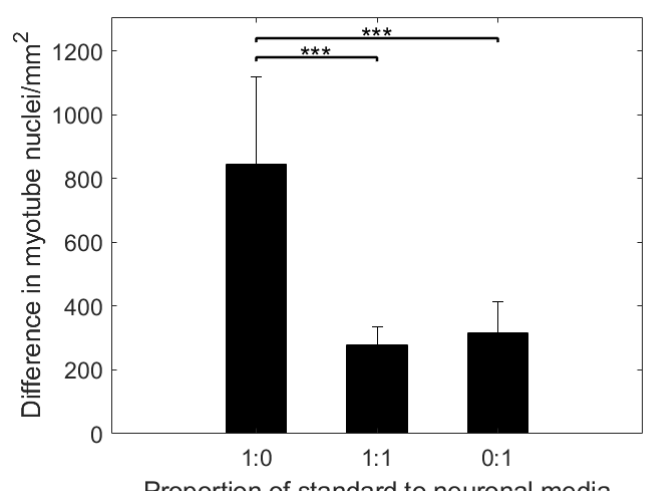

(d)

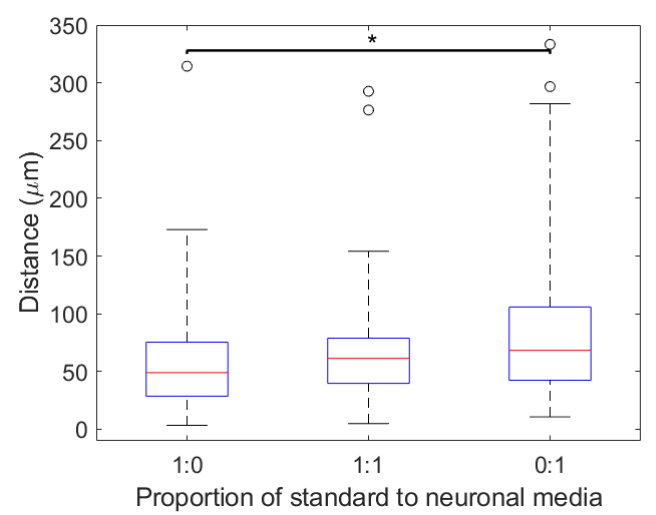

(f)

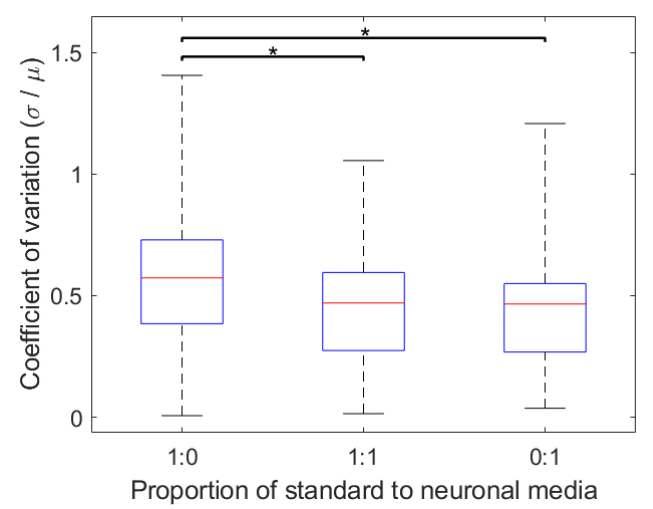

Figure 3. Myotube quality indicators from images of cells in different media compositions at day 5 of differentiation. Difference in myonuclei per $\mathrm{mm} 2$ between days 0 and 5 of differentiation in media with (a) varying serum concentrations and (b) varying proportion of neuronal medium. Mean distance between myonuclei in media with (c) varying serum concentrations and (d) varying proportion of neuronal medium. Uniformity of distance between myonuclei expressed as 'coefficient of variation' in media with (e) varying serum concentrations and (f) varying proportion of neuronal medium. 


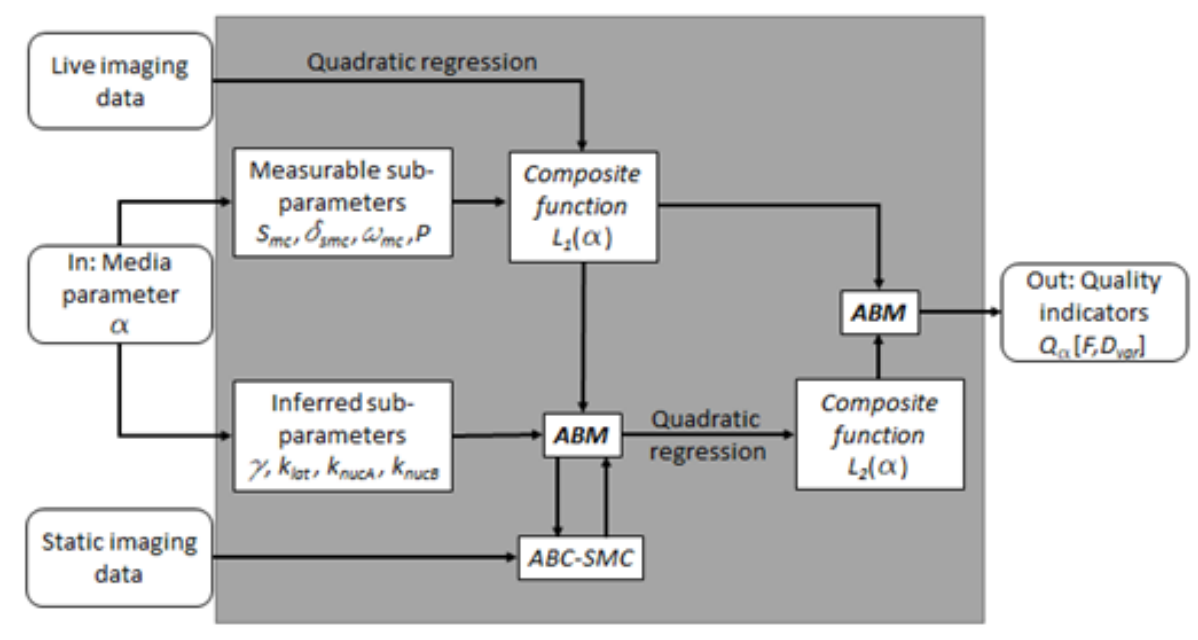

Figure 4. Diagram outlining procedure for conducting virtual experiments using live and static images, an Agent Based Model and Approximate Bayesian Computation.

workflow in creating a predictive model of cell quality is presented in figure 4 . To determine the input ${ }_{203}$ cell behaviours for any combination of differentiation media parameters, a composite function, $\mathrm{M}\left(L_{1}\left(\alpha, \quad{ }^{204}\right.\right.$ $\beta), L_{2}(\alpha, \beta)$ ), was applied where $\alpha$ is the concentration of neuronal medium and $\beta$ the percentage of ${ }_{205}$ horse serum in the culture media. The function $\mathrm{M}$ is informed by two subsets of myoblasts and myotube behavioural functions obtained from in vitro imaging data (described in methods):

1. Function subset $L_{1}(\alpha, \beta)$, composed of functions of observable myoblast behaviour (myoblast speed, standard deviation of speed, standard deviation in angular velocity and chance of cell proliferation per minute) with discrete values derived from live imaging data.

2. Function subset $L_{2}(\alpha, \beta)$, composed of functions of cell behaviours not directly observable but necessary to inform an ABM of myoblast-myotube cell fusion and dispersion.

Once the cell behaviours required for $L_{1}$ and $L_{2}$ (Table 1) were derived for discrete media compositions, ${ }^{213}$ they were interpolated over the parameter space by fitting a second-degree polynomial surface model.

\begin{tabular}{|c|c|}
\hline Model parameter & Observed or inferred from data \\
\hline Myoblast count at $\mathrm{t}_{0}$ & Observed \\
Myotube proportion at $\mathrm{t}_{0}$ & Observed \\
Myoblast speed of motion $\left(S_{m c}\right)$ & Observed \\
Myoblast angular variation $\left(\omega_{m c}\right)$ & Observed \\
Myoblast proliferation chance $\left(P_{m}\right)$ & Observed \\
Nuclei area of influence $($ AoI $)$ & Inferred \\
Residence time threshold $\left(\mathrm{t}_{\text {rmax }}\right)$ & Inferred \\
Coefficient of lateral force $\left(k_{\text {lat }}\right)$ & Inferred \\
Coefficient of nuclei repulsion force $\left(k_{\text {nuc }}\right)$ & Inferred \\
Myotube-myotube fusion time threshold $\left(\mathrm{t}_{\text {MTfuse }}\right)$ & Inferred \\
\hline
\end{tabular}

Table 1. ABM input parameters

The total nuclei count and proportion of myonuclei, required to initialise the ABM, were taken from the mean values of static images from day 0 of differentiation and set to 420 and $53 \%$ respectively. 


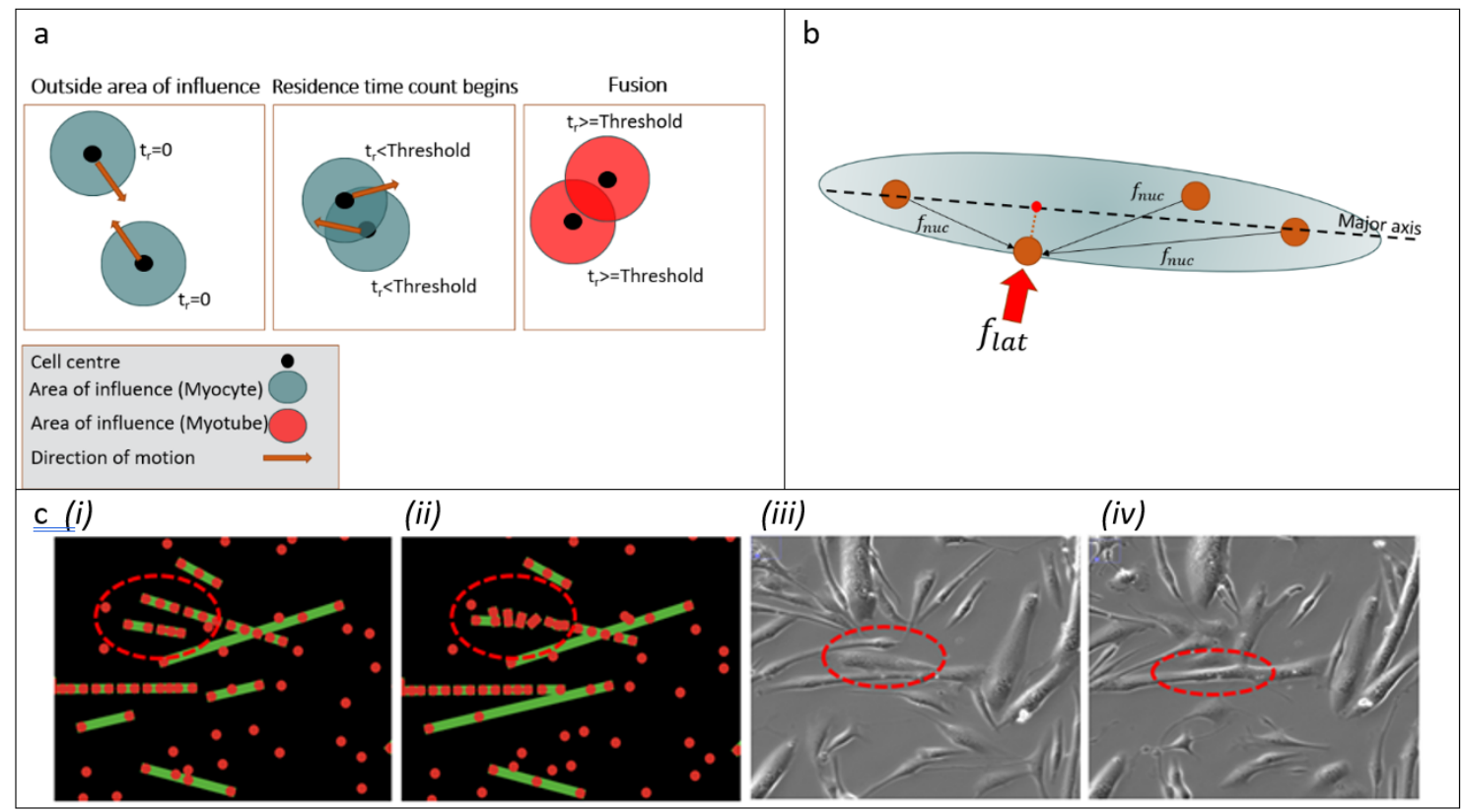

Figure 5. (a) Residence time model of myoblast-myotube fusion. (b) Representation of forces acting on a single nucleus. A lateral force $\left(f_{l} a t\right)$ moves the nucleus towards the major axis of nuclei in a myotube in a direction normal to the major axis. Total nuclei repulsion force is the sum of the individual repulsion forces $\left(f_{n} u c\right)$ acting on the nucleus. (c) Myotube-myotube fusion in agent-based model at (i) 0 minutes and (ii) 15 minutes and in live images at (iii) 0 minutes and (f) 15 minutes.

Modelling of cell fusion events in the ABM is guided by the residence time of two or more cell nuclei when in proximity (Figure 5a). A maximum residence time $\left(\mathrm{t}_{r \max }\right)$ Area of Influence (AoI) surrounding all nuclei were prescribed to all nuclei. When the distance between two nuclei is less than the radius of the AoI, a residence time count $\left(t_{r}\right)$ is initiated for both nuclei. When $t_{r} \dot{i} t_{r \max }$ cleus changes its state from myoblast to myotube, indicating cell fusion. Sensitivity analysis of reasonable values of AoI and $t_{r \max }$ aled a strong linear relation between both parameters in predicting final myonuclei density (supplementary materials $\mathrm{S} 1$ ). Variables $\mathrm{t}_{\operatorname{rmax}}$ and AoI can therefore be condensed into a single variable, $\gamma=A o I / t_{r \max }$ with units $\mu \mathrm{m}^{2} /$ minute. To simplify the choice of parameters, below we fix AoI to a circle with radius of $20 \mu \mathrm{m}$ and vary $\mathrm{t}_{\text {rmax }}$.

To simulate the spatial distribution of myonuclei, a balance of forces acting on nuclei within each myotube was introduced to the ABM. Myonuclei have been shown to initially cluster at the centreline of the cell before spreading out laterally [9] [10. Previous studies by Manhart et al. [17] [18] on spatially constrained nuclei in Drosophila myotubes successfully accounted for nuclei distribution over time by implementing a force balance. Forces considered in this study are (Figure 5b) a lateral force $\left(f_{\text {lat }}\right)$ to simulate the constraints myotube shape and a combined nuclei repulsion force $f_{n u c}$ which simulates the actin-driven lengthwise movement of nuclei throughout the maturation process and any polar forces acting upon nuclei.

By denoting the position of the $\mathrm{i}$-th nucleus at time $\mathrm{t}$ as the $2 \mathrm{D}$ variable $\mathrm{X}_{i}(\mathrm{t})$, as characterised by Manhart et al [17], the velocity of the $i$-th nucleus is equal to the sum of all scalar forces acting upon it:

$$
\begin{gathered}
\frac{d X_{i}}{d t}=\sum_{j \neq i, j=1}^{N} f_{n u c}\left(\left\|X_{i}-X_{j}\right\|\right) \frac{X_{i}-X_{j}}{\left\|X_{i}-X_{j}\right\|}+f_{\text {lat }}\left(\left\|X_{i}-X_{\text {cent }, i}\right\|\right), \\
X_{i}(0)=X_{i}^{0}, i=1,2, \ldots, N
\end{gathered}
$$

Where $\mathrm{X}_{c e n t, i}$ is the point on the major axis of nuclei in a given myotube closest to $\mathrm{X}_{i}$. The nuclei repulsion force can be expressed as: 


$$
f_{n u c}(d)=-k_{n u c}
$$

Where $k_{n u c}$ is a constant defined within the ABM and $d$ is the distance between myonuclei. There is no limit on the distance at which nuclei can interact 18. A similar force with magnitude inversely proportional to distance $d$, as described by Manhart et al was trialled, but was found to tend towards uniformity of nuclei distribution faster than described by our imaging data.

To apply $f_{\text {lat }}$, a linear interpolation is applied to the co-ordinates of nuclei belonging to a given myotube to determine the major axis of the cell. The nuclei are then moved in a direction orthogonal to the major axis towards the centre of the cell with a magnitude:

$$
f_{\text {lat }}\left(d_{\text {lat }}\right)=d_{\text {lat }} k_{\text {lat }}
$$

where $d_{\text {lat }}$ is the shortest distance to the major axis from a given nucleus and $k_{\text {lat }}$ is a constant defined within the ABM. During preliminary ABM simulations, nuclei of neighbouring myotubes were observed fusing together to create a single myotube (Figure 5;(i-ii)). This myotube-myotube fusion replicates observations in vitro (Figure 5s.(ii-iv)) during differentiation. These fusion events continued throughout the entire span of differentiation in the ABM simulations resulting in myotubes containing significantly higher densities of nuclei than were observed in vitro at day 5 and beyond. From this we reason that there are mechanisms which constrain myotube-myotube fusion during the later stages of differentiation and so include a myotube-myotube fusion time threshold $\left(\mathrm{t}_{M T \text { fuse }}\right)$ as a maximum 'age-limit' on fused nuclei after which they cannot fuse with other myonuclei.

The parameters $\gamma, t_{M T f u s e}, k_{l a t}$ and $k_{n u c}$ form function subset $\mathrm{L}_{2}(\alpha, \beta)$. These parameters were derived via statistical inference using an approximate Bayesian computation sequential Monte Carlo (ABC-SMC) method 19]. ABC approximates a posterior distribution for each parameter by comparing the distance between summary statistics provided by the ABM with those measured from experimental images using a distance function $(\delta(x))$. Parameter value $x$ is rejected if the value of $\delta(x)$ exceeds a threshold distance $(\varepsilon)$, building a posterior distribution from accepted parameter values. To speed this process, a sequential Monte-Carlo method was applied to systematically reduce the size of $\varepsilon$ and so converge towards a choice of ABM parameters which best reproduce the experimental observations. An ABC-SMS method is well suited for parallelising, accounts for stochasticity within the ABM and provides information concerning uncertainties in parameter approximation.

The number of fusion events $(F)$, mean coefficient of variance $\left(D_{\text {var }}\right)$ and median myonuclei distance $(D)$ at day 5 of differentiation were chosen as input summary statistics. $D_{v a r}$ is a measure of the uniformity of nuclei distribution but does not describe the magnitude of spatial distribution and so D is required to provide a sufficient measure of nuclei distribution. A distance function was defined as:

$$
\delta\left(F, D, D_{v a r}\right)=\sqrt{\left(\frac{F^{*}-F}{\sigma_{F}}\right)^{2}+\left(\frac{D^{*}-D}{\sigma_{D}}\right)^{2}+\left(\frac{D_{v a r}^{*}-D_{v a r}}{\sigma_{D_{v a r}}}\right)^{2}}
$$

providing the $L^{2}$ norm of differences between results from ABM simulations $\left({ }^{*}\right)$ and data measured from experimental images for $F, D$ and $D_{v a r}$, normalised by the standard deviations of measured data, giving dimensionless residuals for comparison. We assumed the posterior distribution for each parameter to be normal and found the mean and standard deviation as summary statistics for each experimental trial. We then interpolated over the parameter space by fitting a second-degree polynomial surface model.

The posterior for parameter $k_{\text {lat }}$ did not converge towards a fixed normal distribution. This is not surprising, as the arguments of the distance function are not direct indicators of the speed at which nuclei move towards the centreline. Despite this lack of convergence, the posterior for all experiments suggested a range of feasible values for $k_{l a t}$ to be between 0.02 and 0.09 . To represent this within the $\mathrm{ABM}$, a value for $k_{\text {lat }}$ between this range was randomly choosing from a uniform distribution at each required timestep.

ABM simulations produced mean $F$ and $D_{\text {var }}$ and median $D$ within 1 standard deviation of those observed experimentally for most conditions (Supplementary materials S2), implying that the in vitro / 
$\mathrm{ABM}$ workflow is sufficiently calibrated to allow predictions of $\mathrm{Q}$ from a given input media $\alpha$ and $\beta$. The ABM could not reproduce the $\mathrm{F}$ within one standard deviation for simulations of experiments with $2 \%$ serum and 1:1 muscle to neuronal media and $2 \%$ serum and $100 \%$ neuronal medium. This is due to the total number of myoblast fusion events from days 0 to 5 being significantly smaller than the initial number of myoblasts. The ABM assumes that all myoblasts have the potential to fuse throughout the differentiation stage and so the minimum number of fusion events is the total number of nuclei at day 0 . These results suggest that the choice of media may influence the proportion of myoblasts which switch behaviour from proliferation to fusion and that, depending upon the media, a proportion of myoblasts remain in a proliferative state and do not fuse. Defining the proportion of these unfused myoblasts is beyond the scope of this paper but may be useful in future refinements of a predictive model.

\section{Fitting surface models from discrete in-vitro experimental data enables pre- diction of myoblast and myotube behaviours}

Our goal is to create a predictive model of muscle quality based on key early-stage myoblast and myotube cell behaviours. To achieve this, we must first describe how these key behaviours change with respect to the composition of the differentiation media used. We assume that the effects of media composition on cell behaviour, while non-linear, are of a lower order than the complex effects of media composition upon final cell quality. A second-degree polynomial surface model was applied to fit metrics of cell behaviours observed from live imaging $\left(\mathrm{S}_{m c}, \omega_{m c}\right.$, and $\left.\mathrm{P}\right)$ and inferred via the $\operatorname{ABC-SMC}\left(\gamma, \mathrm{t}_{\text {MTfuse }}, k_{\text {lat }}\right.$ and $\left.k_{n u c}\right)$ to provide input cell behaviours for all concentrations of serum and neuronal medium. These predictions of cell behaviour are visualised in figure 6 a-f.

Maximum myoblast speed is projected to be found in media with $3.5 \%$ serum and no neuronal medium present. An additional local maxima is shown to occur in media with $5.5 \%$ serum and $100 \%$ neuronal medium. Variation in myoblast angular velocity shows a general increase in variance (and thus a decrease in cell persistence) with increased serum concentration, with a maximum of 23 degrees per minute occurring in media with $7.5 \%$ serum and $100 \%$ neuronal medium. A maximum chance of proliferation of $1.1 \times 10^{-3}$ per cell per minute is expected for media with $5 \%$ serum and $100 \%$ neuronal medium.

Increasing serum concentration was shown to correspond with an increase in the maximum residence time threshold (Figure 6d) suggesting that, at higher serum concentrations, myoblasts require a longer time in contact with myotubes before fusion can occur. The coefficient of nuclei repulsion force $\left(k_{n u c}\right)$, (Figure 6e) showed a general trend for increasing with increase in serum concentrations, with a slight decline at high concentrations of serum $(>\sim 7 \%)$. For media with lower serum concentrations $(<\sim 4 \%)$, $k_{n u c}$ increases as the concentration of neuronal medium is increased.

The maximum age at which two or more myotubes may fuse together was projected to be between 3 to 4.5 days of cell differentiation for most media conditions (Figure 6). This is in agreement with observations of actin striations occuring in cells after day 3. Myotube-myotube fusion was shown to end earlier in cells with increased levels of neuronal medium and continue for longer in cells with higher serum concentrations. Since simulations were ended at day 5 , cells in a medium with no neuronal medium and $10 \%$ (or greater) serum concentration may still allow fusion at day 5 and beyond.

Parameters of the observed and inferred cell behaviours studied were combined to provide input parameters to the composite function $\mathrm{M}\left(L_{1}(\alpha, \beta), L_{2}(\alpha, \beta)\right)$, describing cell and nuclei behaviour in culture media with any concentration of neuronal medium and serum. A parameter sweep was conducted through values of a from 0 to $100 \%$ neuronal medium and $\mathrm{b}$ from 0 to $10 \%$ serum concentration, with early indicators of muscle cell quality $Q\left[F, D, D_{v a r}\right]$ as an output. Outputs were obtained from averaging over 10 simulations for each parameter set to account for stochasticity within the model.

\section{Difference in total myonuclei production is inversely related to early-stage nuclei fusion index}

Conditions generating small (300-400 nuclei $\left./ \mathrm{mm}^{2}\right)$ total increases in myonuclei by day 5 (Figure 7 a) exhibit a negative exponential curve in the increase in nuclei density over time and a steep initial decline 
(a)

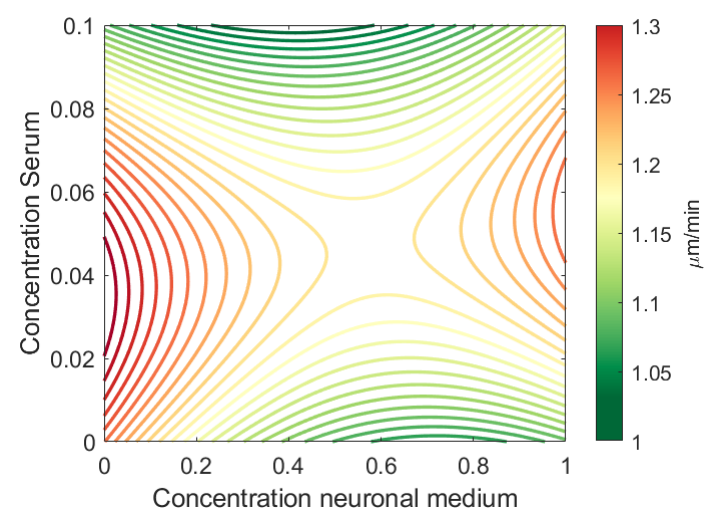

(c)

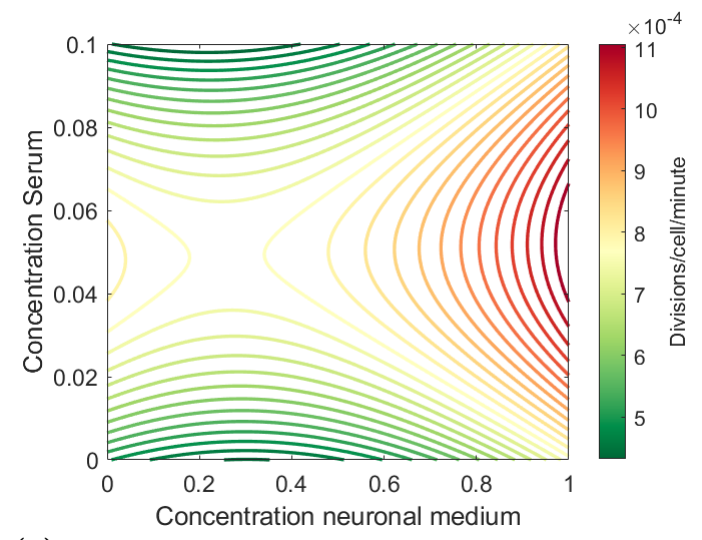

(e)

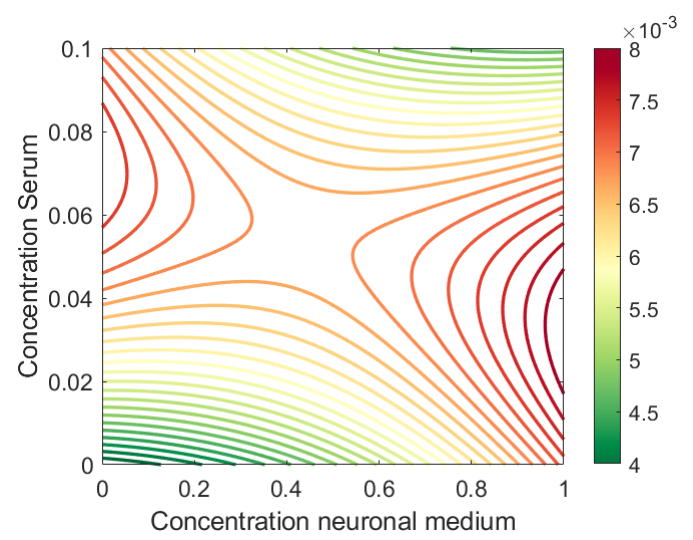

(b)

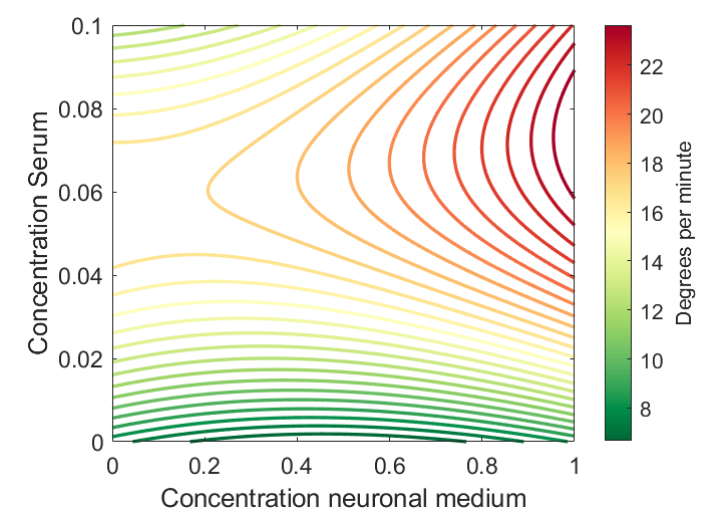

(d)

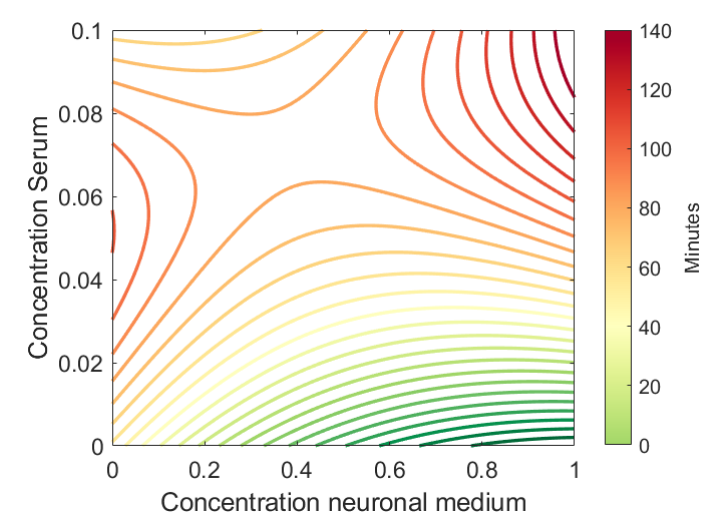

(f)

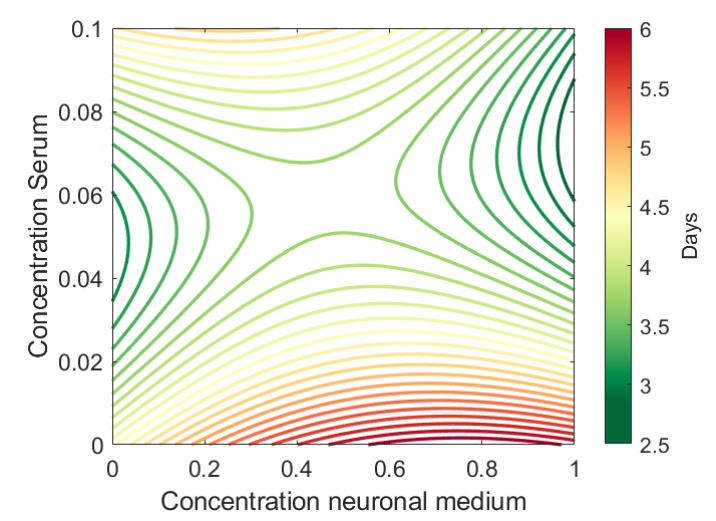

Figure 6. Fitted surface models for cell and nuclei observed (a-c) and inferred (d-f) behavioural parameters. (a) Speed of myoblast motion, (b) variation in myoblast angular velocity, (c) myoblast chance of proliferation, (d) residence time threshold, (e) nuclei repulsion force coefficient and (f) maximum age at which myoblasts can fuse. 
(a)

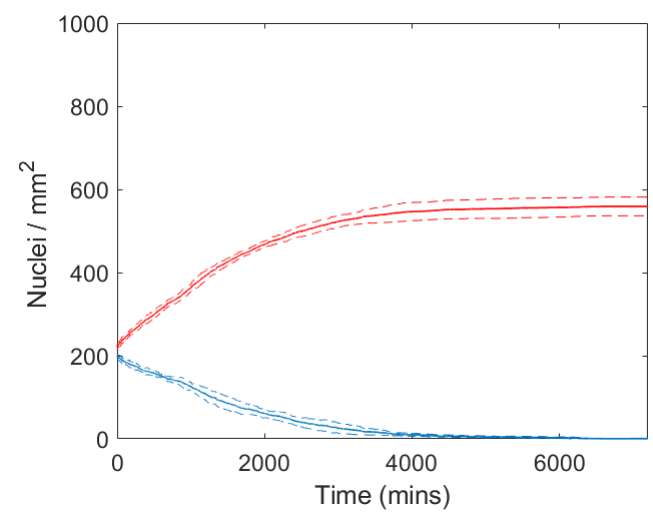

(b)

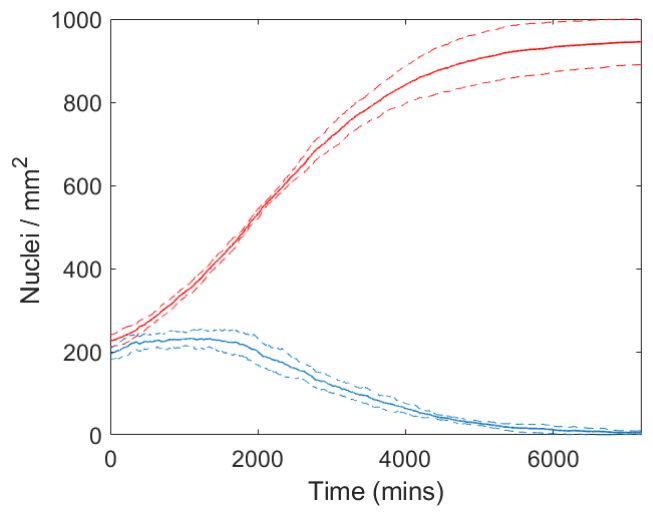

(c)

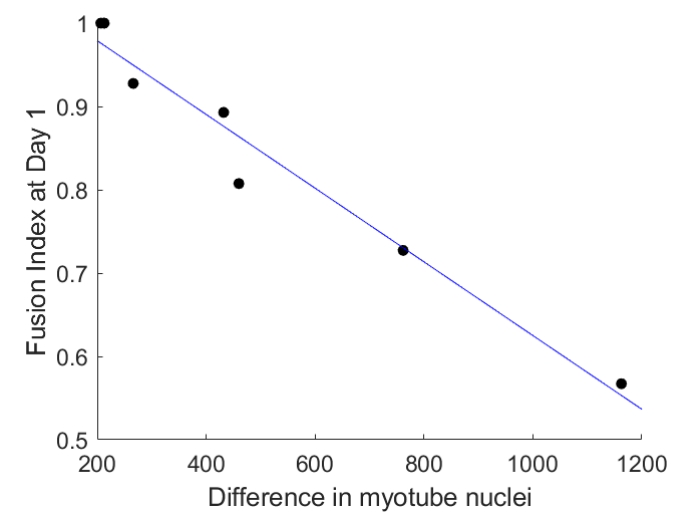

Figure 7. Examples of ABM generated changes in myotube (red) nuclei and myoblast (blue) nuclei counts over time for conditions (a) $0 \%$ serum in neuronal medium and (b) $2 \%$ serum in neuronal medium (dashed lines represent standard deviation over 5 runs) and (c) ABM generated differences in number of myonuclei between days 0 and 5 against fusion index $\left(\mathrm{R}^{2}=0.96\right)$.

in myoblast numbers while larger total increases in myonuclei (Figure $7 \mathrm{~b}$ ) are associated with a sigmoidal increase in myonuclei over time with nuclei initially increasing or remaining stable before decreasing. To account for these distinctive trends, we first compared the rate of myonuclei production between days 0-1 with day 5 difference in myonuclei for all media type experiments but found no significant correlation.

Further analysis indicates that these differences in total number of myonuclei (and therefore the number of fusion events) relate to the ratio of myonuclei to total nuclei, described by the nuclei fusion index [20], during the initial stages of differentiation. There is a strong negative linear relation $\left(\mathrm{R}^{2}=0.96\right)$ between day 0 - day 5 difference in myonuclei density and the nuclei fusion index at day 1 (Figure 7 F). This suggests that conditions which provide a high fusion index in the early stages of differentiation will result in a lower number of fusion events overall and vice-versa. Practically, this implies a trade-off between the magnitude of the fusion index (frequently used as an indicator of experimental efficiency) and the final quantity of muscle cells.

\section{ABM simulations predict a compromise between cell quantity and quality when selecting optimal culture media composition}

Our model predicts that difference in myotube nuclei density at day 5, an indicator of total cell fusion, initially increases as serum concentration increases (Figure 8a). We also predict a general reduction in the difference in myotube nuclei density with increasing proportion of neuronal medium. These effects 
(a)

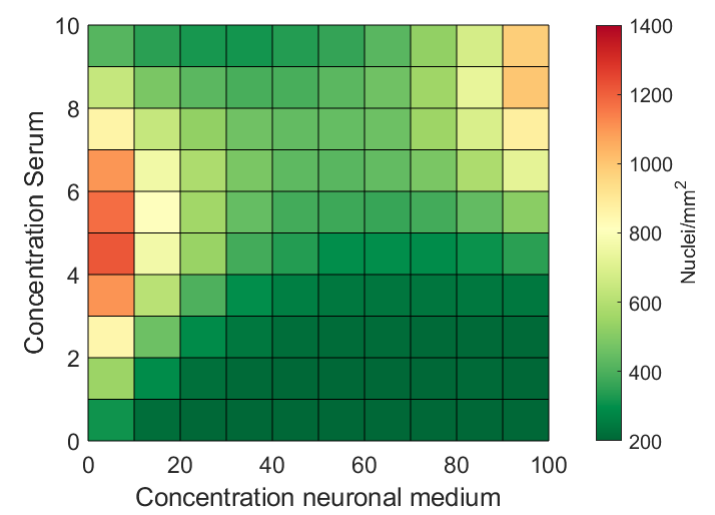

(c) (b)

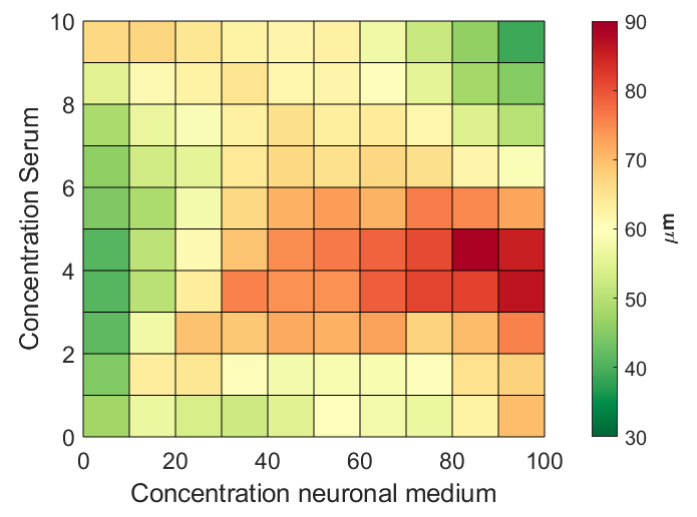

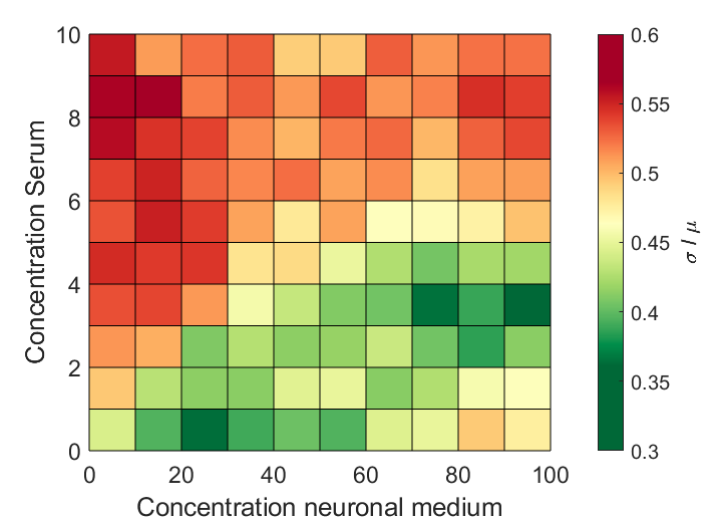

Figure 8. Surface plots showing predicted quality indicators over a range of media conditions. (a) Difference in number of myonuclei between day 0 and day 5, (b) Mean distance between myonuclei at day 5 and (c) coefficient of variation of myonuclei at day 5 .

were shown to be non-linear, with maximum total fusion events predicted at serum concentrations of $4-5 \%$ and no neuronal medium, with a second local maxima for $100 \%$ neuronal medium with $8-9 \%$ serum concentration. A region of low total cell fusion was observed for media containing neuronal medium with serum concentrations below $4 \%$. The higher total fusion at serum concentrations above this region suggests that increasing serum concentration may compensate for the reduction in muscle cell quantity caused by the presence of neuronal medium. It should be noted that the inverse relation between fusion index and difference in myonuclei described above indicates that media conditions showing a large difference in nuclei will have a lower fusion index during the crucial first days of differentiation.

Predictions of the coefficient of variation (Figure 8k), show a decrease in the uniformity of nuclei distribution as the concentration of serum increases and thus a predicted decrease in cell quality. Nuclei uniformity did not decrease with increasing neuronal medium, indeed there are regions in which uniformity, and thus cell quality, is increased. The lowest variation in nuclei distribution was shown to be in $100 \%$ Neuronal medium with $3-4 \%$ serum concentration with a further local minima at $20-30 \%$ neuronal medium and no serum. Increasing the proportion of neuronal medium was shown to increase the mean distance between myotube nuclei (Figure 8b) with a maximum at a media composition of $4-5 \%$ serum and 80-90\% neuronal medium, corresponding to a region with low total fusion but high spatial uniformity.

Taken together, these results predict that selecting a media composition for optimum muscle cell production presents a trade-off between the quantity and quality of cells produced. Increasing serum concentration tends to increase cell quantity but decreases quality, while increasing the proportion of neuronal medium can result in significant reductions in cell quantity but increased quality. 
(a)

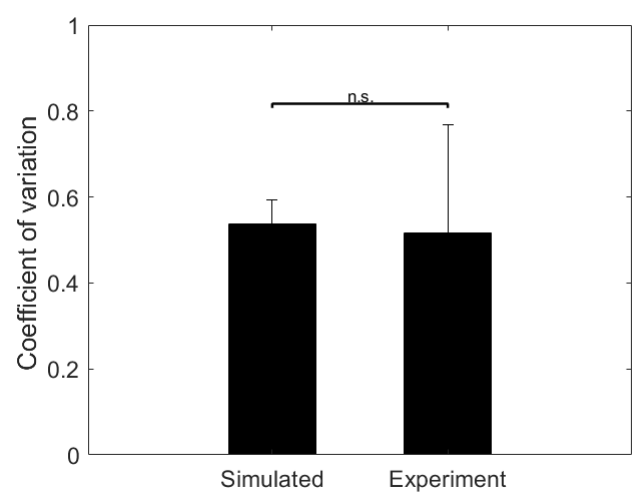

(b)

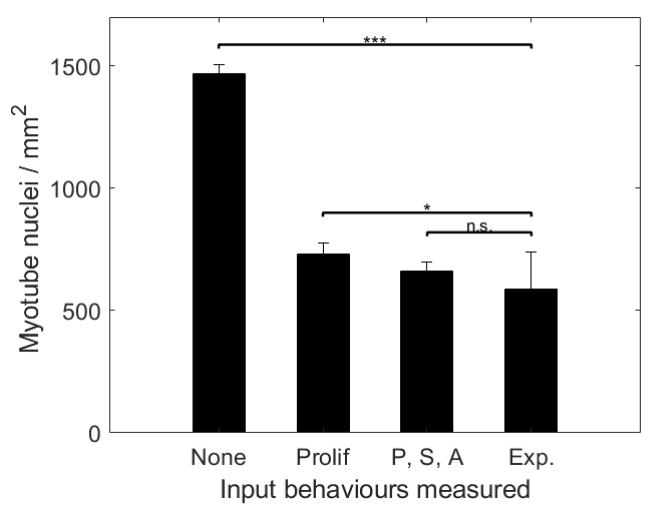

Figure 9. Comparison of simulated outputs of quality indicators against values measured from in vitro images. (a) Coefficient of variation (a measure of uniformity) in myonuclei. (b) Difference in myonuclei between day 0 and day 5 for simulations with (from left to right) all inputs taken from

\section{$\mathrm{ABM}$ simulations predict cell quality in $5 \%$ serum}

Surface fitting of cell behavioural metrics over all media compositions (figure 6) predicts local extremes in behaviours in media with $5 \%$ serum and $100 \%$ muscle differentiation medium. This media composition was chosen to validate the predictions of the ABM model. Paired t-tests indicate that there is no significant difference between ABM predictions and in vitro measurements for the coefficient of variation in myonuclei (figure 92), suggesting the simulations replicate uniformity of nuclei distribution.

A significant difference was observed between ABM predictions of the difference in myonuclei density and in vitro observations (figure $9 \mathrm{~b}$ ). To ascertain potential sources of model discrepancies, live images of cells in $5 \%$ serum with muscle differentiation medium were analysed to observe cell behaviours and compare with those derived from surface fitting. Measured angular variation and speed of cell motion exhibited modest deviations between fitted and measured (mean absolute error of 3.6 degrees/minute and $0.13 \mu \mathrm{m} /$ minute respectively), while cell proliferation rate was significantly lower than predicted (Normalised mean absolute error of 110\%). Exchanging the measured cell proliferation rate rather than the fitted value as an input to the ABM significantly reduced the difference between simulated and observed differences in myonuclei (figure 9b). The additional application of measured values speed and angular variation further decreased the difference but with a much smaller effect. This indicates that the modelling workflow described here can make predictions on the difference in myonuclei when initial cell proliferation rate is known. These predictions improve as more initial cell behaviour metric values are known.

\section{Discussion}

Optimisation of cell culturing protocols in tissue engineering is essential for maintaining the quantity and functionality of mature tissue as well as ensuring experimental reproducibility. The move towards replicating in vivo tissues and organs requires co-culturing of cell types, presenting a challenge for traditional protocols designed for cell monocultures as we seek environments in which multiple cell types can thrive. Applying quantitative mathematical and computational modelling approaches alongside in vitro experiments increases efficiency and reduces costs when designing cell culturing procedures, aiding the transition from bench to bedside for regenerative medicine 21].

Here, we focus on culturing muscle cells from murine myoblasts, optimising concentrations of serum and neuron differentiation medium with the aim of sustaining a co-culture of muscle cells and motor neurons. To achieve this, we employed metrics for quantifying the migratory behaviour of myoblasts and developed novel metrics for indicating the quality and quantity of mature cells. This allowed us to 
quantify a full spectrum of cell behaviours from myoblasts to myotubes throughout the differentiation period.

We found that varying the concentrations of media components produces significant changes in indicators of mature muscle cell quality and key early-stage cell behaviours. Describing metrics of the cell behaviours as a function of media composition allowed us to create an agent-based model with the ability to predict final cell quality for any given concentration of serum or neuron differentiation medium.

Combined in vitro - in silico experiments indicate that choice of media composition presents a balance between cell yield and cell quality. We show that increasing the concentration of serum in cell differentiation medium increases the total amount of myoblast fusion but has a detrimental effect on cell quality. We also show that cell quality can be maintained (or even improved) by the addition of neuronal differentiation medium. While this is encouraging for the potential co-culturing of skeletal muscle and motor neurons, it comes at the cost of lower myoblast fusion when compared to muscle differentiation medium alone.

Uniform distribution of nuclei is known to be associated with healthy muscle tissue [22], with aggregation of nuclei linked to muscular disfunction 9 23] 24]. In this study, the mean distance between nuclei and coefficient of variation of nuclei were defined as metrics of the uniformity of myonuclei distribution within a cell. The difference in myonuclei density per $\mathrm{mm}^{2}$ over the first 5 days of differentiation was used as a second indicator of cell outcomes. As well as a marker of the potential yield of muscle cells, difference in myonuclei density was found to have a strong negative linear relation with initial fusion index, a widely used indicator of the effectiveness of cell fusion during differentiation.

Serum is added to media to stabilise conditions for cell proliferation though differentiation in some muscle cell lines has been shown to be triggered by the deprivation of serum. Serum represents an unknown in terms of composition large batch-to-batch as well as source-to-source variability, which may alter motor neuron differentiation efficiency and trigger loss of stemness. Our results show that an increase in media serum percentage leads to increasingly non-uniform distributions of nuclei within myotubes, a sign of less viable muscle cells. These findings agree with previous work by Lawson and Purslow [25] which shows C2C12 cells differentiated best in low serum or serum-free media and other studies 26] which show using media with low or no serum is advantageous in preparation for co-culturing muscle cells with iPS derived motor neurons. Our work, however, indicates that the relationship between serum concentration and cell fusion is non-linear, and that increasing serum to moderate levels (up to around 5\%) increases the total amount of cell fusion. This could enable a strategy of adding more serum to muscle cells cultured in neuronal differentiation media to boost cell quantity without impacting quality.

Trials showing greater increases in myotube nuclei density at the end of the differentiation phase exhibited a lower fusion index during the early stages of differentiation. This appears counter-intuitive, but since our simulations indicate that myoblast availability is a limiting factor in cell fusion rate, we hypothesize that media compositions with a lower fusion index prioritise myoblast proliferation over fusion during the early stages of differentiation. The supply of myoblasts will therefore not be exhausted as quickly as more fusion-efficient regimes, leading to a greater total amount of fusion. The negative relation between fusion index and total amount of fusion has wider implications for the reliance of fusion index alone as a marker of experimental success. As fusion index measures the ratio of myonuclei to mononucleated myoblasts at a given time, it conflates the rate of cell-cell fusion with the proliferation rate of myoblasts. While fusion index is simple to calculate and gives an intuition of initial experimental efficiency, it is important to consider that a high fusion index may be due to a high fusion rate of neighbouring cells or a low rate of background cell division, or some more complex balance between the two.

Since availability of myoblasts appears to be a limiting factor in the total amount of fusion, and thus the final volume of cells, it may seem intuitive to culture cells to confluence during the growth phase so there is greater availability. Previous studies 27. however, show that seeding myoblasts at a high density induces quiescence in cells and suppresses differentiation and so would not provide an effective strategy. Committed myoblasts need to switch from a proliferation state (growth phase) to a fusion state (differentiation phase). In in vitro culture, this switch is triggered by lowering media serum content which causes myoblasts to initiate cell cycle arrest and differentiate into myotubes, but this behaviour 
may be strongly altered if medium composition and serum percentage are not optimal. Calibration of our ABM highlighted a significant difference between measured cell proliferation rates and those predicted by a surface fitting model and from previous findings [25] that the cell proliferative state is preserved at higher serum concentrations. Our work suggests that the effects of media composition on switching cell states are complex and warrant further investigation. It also indicates that the prognostic accuracy of the ABM increases as more of the cell behaviours are understood. The workflow described here is intended as an iterative process between in vitro and in silico experiments.

Our numerical modelling assumes that metrics of cell motion do not change over time. It may be expected that cell migration speed and angular variation change as myotube density increases with time though we found no significant changes in metrics of behaviour (results not shown) throughout the first day of differentiation. Temporal changes over a longer timescale were not studied but assumed to have a minor impact on model outputs due to the short timespan ( $1-3$ days) in which the bulk of fusion occurs. Our model also assumes that all myoblasts may differentiate into myotubes. C2C12 cells which are deficient in the production of the proteins Myf-5 and Myo-D have been shown to fail to form myotubes in culture 28]. The low final myonuclei densities observed here for high levels of neuron differentiation media indicate that not all myoblasts are viable. Nuclei staining which differentiate between myoblasts and myonuclei would allow a clearer observation of myoblast fates under varying media conditions and allow for the quantification of non-fusing myoblasts into the model. Additional early markers of cell quality include myotube width and the presence of actin striations in myotubes [29]. We found no significant differences in width and a high proportion of actin striations present in all trials and so did not include them in our investigation though they may be relevant for optimising other cell culture protocols or cell lines.

Previous work 25 has shown that the response of cell differentiation to serum concentration is cell line dependent. We based our experimentation on the use of primary murine myotubes to identify and extract crucial parameters needed to fine tune media conditions for co cultures of myotubes and motor neurons. This quantitative assessment may also be applied to assess cell quality for artificial muscles generated from human iPS, allowing to optimize culture and media conditions which steer cell linage. Ultimately, adding motor neurons to innervate the cells would allow to generate organ-on-chip platforms that successfully recapitulate human NMJs in vitro suitable for disease modelling.

In summary, we identified new quality indicators for muscle cells, which can be implemented in future statistical approaches to optimisation and showed that changes in the composition of muscle cell differentiation media affect both early-stage cell migratory behaviours, cell fusion index and final cell quality. By representing early-stage cell behaviours as a function of media composition, we designed an $\mathrm{ABM}$ to predict cell quality for any given configuration of media. Our results indicate that the choice of culture media composition will necessarily involve a compromise between the quality of cells and total cell yield. The iterative workflow of in vitro - in silico experiments presented here can be applied as a tool for the optimisation of a wide range of further muscle cell culturing parameters and muscle progenitor cell lines, including human iPS, providing an efficient and cost-effective method for improving and quantifying tissue engineering procedures.

\section{References}

1. Bhatia, S. N. \& Ingber, D. E. Microfluidic organs-on-chips. Nature Biotechnology 2014 32:8 32, 760-772 (2014).

2. Zamprogno, P., Wüthrich, S., Achenbach, S., Thoma, G., Stucki, J. D., Hobi, N., Schneider-Daum, N., Lehr, C.-M., Huwer, H., Geiser, T., Schmid, R. A. \& Guenat, O. T. Second-generation lungon-a-chip with an array of stretchable alveoli made with a biological membrane. Communications Biology 2021 4:1 4, 1-10 (2021).

3. Lind, J. U., Busbee, T. A., Valentine, A. D., Pasqualini, F. S., Yuan, H., Yadid, M., Park, S.-J., Kotikian, A., Nesmith, A. P., Campbell, P. H., Vlassak, J. J., Lewis, J. A. \& Parker, K. K. Instrumented cardiac microphysiological devices via multimaterial three-dimensional printing. Nature Materials 2016 16:3 16, 303-308 (2016). 


\begin{tabular}{|c|c|c|}
\hline Name & Description & Symbol \\
\hline Media function & $\begin{array}{l}\text { General term for function determining quality in- } \\
\text { dicators for a given media parameter. }\end{array}$ & $M(x)$ \\
\hline $\begin{array}{l}\text { Proportion of neuronal } \\
\text { medium }\end{array}$ & $\begin{array}{l}\text { The fraction of differentiation medium comprised } \\
\text { of neuron specific N2B } 27 \text { medium. }\end{array}$ & $\alpha$ \\
\hline Serum concentration & $\begin{array}{l}\text { Percentage of serum by volume in cell culture } \\
\text { media. }\end{array}$ & $\beta$ \\
\hline Quality indicators & $\begin{array}{l}\text { A set of measurable early indicators of eventual } \\
\text { muscle cell quality and quantity. }\end{array}$ & $Q$ \\
\hline Fusion events & $\begin{array}{l}\text { Cumulative count of myoblast-myoblast and } \\
\text { myoblast-myotube fusion events per } \mathrm{mm}^{2} \text { over } \\
\text { a given time } \mathrm{T} \text { (days). }\end{array}$ & $F_{T}$ \\
\hline Myonuclei distance & Distance between myonuclei in given sample cell. & $D$ \\
\hline Coefficient of variance & $\begin{array}{l}\text { Quality indicator of uniformity in myonuclei dis- } \\
\text { tribution }\left(D_{\sigma} / \bar{D}\right) \text {. }\end{array}$ & $D_{\text {var }}$ \\
\hline $\begin{array}{l}\text { Measured myoblast parame- } \\
\text { ters }\end{array}$ & $\begin{array}{l}\text { Set of functions of parameters of myoblast be- } \\
\text { haviour quantified from live imaging data for a } \\
\text { given media parameter. }\end{array}$ & $L_{1}(x)$ \\
\hline Inferred myoblast parameters & $\begin{array}{l}\text { Set of functions of parameters of myoblast be- } \\
\text { haviour inferred from static imaging data and } \\
\text { simulation for a given media parameter. }\end{array}$ & $L_{2}(x)$ \\
\hline Myoblast speed & $\begin{array}{l}\text { Mean product of distance travelled by a myoblast } \\
\text { cell between frames and frame rate }(\mu \mathrm{m} / \mathrm{min}) \text {. }\end{array}$ & $S_{m c}$ \\
\hline Myoblast angular velocity & $\begin{array}{l}\text { Standard deviation of angular deviation of my- } \\
\text { oblast motion between frames multiplied by frame } \\
\text { rate (Degrees/min). }\end{array}$ & $\omega_{m c}$ \\
\hline $\begin{array}{l}\text { Myoblast chance of prolifera- } \\
\text { tion }\end{array}$ & Chance of cell division per cell per minute. & $P$ \\
\hline Area of influence & $\begin{array}{l}\text { Area surrounding nuclei in which the residence } \\
\text { time counter is activated if another nuclei is de- } \\
\text { tected. }\end{array}$ & AoI \\
\hline Residence time threshold & $\begin{array}{l}\text { The maximum time that two nuclei may spend } \\
\text { within each other's AoI before fusion is initiated. }\end{array}$ & $t_{\text {rmax }}$ \\
\hline $\begin{array}{l}\text { Combined residence time pa- } \\
\text { rameter }\end{array}$ & $\mathrm{AoI} / t_{r \max }$ & $\gamma$ \\
\hline $\begin{array}{l}\text { Myotube-myotube fusion time } \\
\text { threshold }\end{array}$ & $\begin{array}{l}\text { The maximum age that a fused nuclei can initiate } \\
\text { fusion with another myonuclei. }\end{array}$ & $t_{M T f u s e}$ \\
\hline Nuclei lateral force constant & $\begin{array}{l}\text { Constant governing the distance-dependent lateral } \\
\text { force enacting on myonuclei. }\end{array}$ & \\
\hline $\begin{array}{l}\text { Nuclei repulsion force con- } \\
\text { stant. }\end{array}$ & $\begin{array}{l}\text { Constant of myonuclei repulsion force, simulating } \\
\text { the actin-driven lengthwise repulsion of nuclei. }\end{array}$ & $k_{n u c}$ \\
\hline
\end{tabular}

Table 2. List of abbreviations

4. Beckwitt, C. H., Clark, A. M., Wheeler, S., Taylor, D. L., Stolz, D. B., Griffith, L. \& Wells, A. ${ }^{503}$ Liver 'organ on a chip'. Experimental Cell Research 363, 15-25 (2018).

5. Mueller, C., Trujillo-Miranda, M., Maier, M., Heath, D. E., O'Connor, A. J. \& Salehi, S. Effects of External Stimulators on Engineered Skeletal Muscle Tissue Maturation. Advanced Materials Interfaces 8, 2001167 (2021).

6. Hindi, L., McMillan, J., Afroze, D., Hindi, S. \& Kumar, A. Isolation, Culturing, and Differentiation ${ }_{508}$ of Primary Myoblasts from Skeletal Muscle of Adult Mice. Bio-Protocol 7 (2017). 
7. Bakooshli, M. A., Lippmann, E. S., Mulcahy, B., Iyer, N., Nguyen, C. T., Tung, K., Stewart, B. A., 510 Van Den Dorpel, H., Fuehrmann, T., Shoichet, M., Bigot, A., Pegoraro, E., Ahn, H., Ginsberg, H., 511 Zhen, M., Ashton, R. S. \& Gilbert, P. M. A 3d culture model of innervated human skeletal muscle 512 enables studies of the adult neuromuscular junction. eLife 8 (2019).

8. Goers, L., Freemont, P. \& Polizzi, K. M. Co-culture systems and technologies: Taking synthetic biology to the next level. Journal of the Royal Society Interface 11 (2014).

9. Roman, W. \& Gomes, E. R. Nuclear positioning in skeletal muscle. Seminars in Cell and Developmental Biology 82, 51-56 (2018).

10. Cadot, B., Gache, V. \& Gomes, E. R. Moving and positioning the nucleus in skeletal muscle-one step at a time. Nucleus 6, 373-381 (2015).

11. Pimentel, M. R., Falcone, S., Cadot, B. \& Gomes, E. R. In vitro differentiation of mature myofibers for live imaging. Journal of Visualized Experiments 2017, 55141 (2017).

12. Maury, Y., Côme, J., Piskorowski, R. A., Salah-Mohellibi, N., Chevaleyre, V., Peschanski, M., Martinat, C. \& Nedelec, S. Combinatorial analysis of developmental cues efficiently converts human pluripotent stem cells into multiple neuronal subtypes. Nature Biotechnology 33, 89-96 (2015).

13. Vis, M. A. M., Ito, K. \& Hofmann, S. Impact of Culture Medium on Cellular Interactions in in vitro Co-culture Systems. Frontiers in Bioengineering and Biotechnology 8, 911 (2020).

14. Möller, J. \& Pörtner, R. in New Insights into Cell Culture Technology (InTech, 2017).

15. Yu, J. S. \& Bagheri, N. Modular microenvironment components reproduce vascular dynamics de novo in a multi-scale agent-based model. Cell Systems 12, 795-809.e9 (2021).

16. Klinger, E., Rickert, D. \& Hasenauer, J. PyABC: Distributed, likelihood-free inference. Bioinformatics 34, 3591-3593 (2018).

17. Manhart, A., Windner, S., Baylies, M. \& Mogilner, A. Mechanical positioning of multiple nuclei in muscle cells. PLoS Computational Biology 14 (ed Igoshin, O. A.) e1006208 (2018).

18. Manhart, A., Azevedo, M., Baylies, M. \& Mogilner, A. Reverse-engineering forces responsible for dynamic clustering and spreading of multiple nuclei in developing muscle cells. Molecular Biology of the Cell 31, 1802-1814 (2020).

19. Schälte, Y. \& Hasenauer, J. Efficient exact inference for dynamical systems with noisy measurements using sequential approximate Bayesian computation. Bioinformatics 36, I551-I559 (2020).

20. Sun, Y., Ge, Y., Drnevich, J., Zhao, Y., Band, M. \& Chen, J. Mammalian target of rapamycin regulates miRNA-1 and follistatin in skeletal myogenesis. Journal of Cell Biology 189, 1157-1169 (2010).

21. Waters, S. L., Schumacher, L. J. \& El Haj, A. J. Regenerative medicine meets mathematical modelling: developing symbiotic relationships. npj Regenerative Medicine 6 (2021).

22. Bruusgaard, J. C., Liestøl, K., Ekmark, M., Kollstad, K. \& Gundersen, K. Number and spatial distribution of nuclei in the muscle fibres of normal mice studied in vivo. Journal of Physiology 551, 467-478 (2003).

23. Metzger, T., Gache, V., Xu, M., Cadot, B., Folker, E. S., Richardson, B. E., Gomes, E. R. \& Baylies, M. K. MAP and kinesin-dependent nuclear positioning is required for skeletal muscle function. Nature 484, 120-124 (2012).

24. Gimpel, P., Lee, Y. L., Sobota, R. M., Calvi, A., Koullourou, V., Patel, R., Mamchaoui, K., Nédélec, F., Shackleton, S., Schmoranzer, J., Burke, B., Cadot, B. \& Gomes, E. R. Nesprin-1 $\alpha$-Dependent Microtubule Nucleation from the Nuclear Envelope via Akap450 Is Necessary for Nuclear Positioning in Muscle Cells. Current Biology 27, 2999-3009.e9 (2017).

25. Lawson, M. A. \& Purslow, P. P. Differentiation of myoblasts in serum-free media: Effects of modified media are cell line-specific. Cells Tissues Organs 167, 130-137 (2000). 
26. Brewer, G. J., Torricelli, J. R., Evege, E. K. \& Price, P. J. Optimized survival of hippocampal 556 neurons in B27-supplemented neurobasal ${ }^{\mathrm{TM}}$, a new serum-free medium combination. Journal of 557 Neuroscience Research 35, 567-576 (1993).

27. Chowdhury, S. R., Muneyuki, Y., Takezawa, Y., Kino-oka, M., Saito, A., Sawa, Y. \& Taya, M. Growth and differentiation potentials in confluent state of culture of human skeletal muscle myoblasts. Journal of Bioscience and Bioengineering 109, 310-313 (2010).

28. Yoshiko, Y., Hirao, K., Sakabe, K., Seiki, K., Takezawa, J. \& Maeda, N. Autonomous control of expression of genes for insulin-like growth factors during the proliferation and differentiation of C2C12 mouse myoblasts in serum-free culture. Life Sciences 59, 1961-1968 (1996).

29. Dwyer, J., Iskratsch, T. \& Ehler, E. Actin in striated muscle: Recent insights into assembly and ${ }_{565}$ maintenance. Biophysical Reviews 4, 17-25 (2012). 